\title{
CR-INVARIANCE OF THE STEINNESS INDEX
}

\author{
JIHUN YUM
}

\begin{abstract}
We characterize the Diederich-Fornaess index and the Steinness index in terms of a special 1-form, which we call D'Angelo 1-form. We then prove that the Diederich-Fornaess and Steinness indices are invariant under CR-diffeomorphisms by showing CR-invariance of D'Angelo 1-forms.
\end{abstract}

\section{Introduction}

Let $\Omega \subset \mathbb{C}^{n}(n \geq 2)$ be a bounded domain with $C^{1}$-smooth boundary. A $C^{1}$-smooth function $\rho$ defined on a neighborhood $V$ of $\bar{\Omega}$ is called a (global) defining function of $\Omega$ if $\Omega=\{z \in V: \rho(z)<0\}$ and $d \rho(z) \neq 0$ for all $z \in \partial \Omega$. The Diederich-Forncess exponent of $\rho$ is defined by

$$
\eta_{\rho}:=\sup \left\{\eta \in(0,1):-(-\rho)^{\eta} \text { is strictly plurisubharmonic on } \Omega\right\} .
$$

If there is no such $\eta$, then we define $\eta_{\rho}=0$. The Diederich-Forncess index of $\Omega$ is defined by

$$
D F(\Omega):=\sup \eta_{\rho}
$$

where the supremum is taken over all defining functions $\rho$. We say that the DiederichFornæss index of $\Omega$ exists if $D F(\Omega) \in(0,1]$. If $D F(\Omega)$ exists, then there exists a bounded strictly plurisubharmonic exhaustion function on $\Omega$. In other words, $\Omega$ becomes a hyperconvex domain. In 1977, Diederich and Fornæss ([5]) proved that for a bounded pseudoconvex domain $\Omega, C^{2}$-smoothness of $\partial \Omega$ implies the existence of $D F(\Omega)$.

In [10], the author introduced the concept of Steinness index which is an analogue of the Diederich-Fornaess index but related to Stein neighborhood bases. The Steinness exponent of $\rho$ is defined by

$$
\begin{array}{r}
\widetilde{\eta}_{\rho}:=\inf \left\{\widetilde{\eta}>1: \rho^{\widetilde{\eta}} \text { is strictly plurisubharmonic on } \bar{\Omega}^{\complement} \cap U\right. \\
\text { for some neighborhood } U \text { of } \partial \Omega\},
\end{array}
$$

where $\bar{\Omega}^{\complement}:=\mathbb{C}^{n} \backslash \bar{\Omega}$. If there is no such $\widetilde{\eta}$, then we define $\widetilde{\eta}_{\rho}=\infty$. The Steinness index of $\Omega$ is defined by

$$
S(\Omega):=\inf \widetilde{\eta}_{\rho}
$$

where the infimum is taken over all defining functions $\rho$. We say that the Steinness index of $\Omega$ exists if $S(\Omega) \in[1, \infty)$. $\bar{\Omega}$ is said to have a Stein neighborhood basis if for any neighborhood $V_{1}$ of $\bar{\Omega}$, there exists a pseudoconvex domain $V_{2}$ such that $\bar{\Omega} \subset V_{2} \subset V_{1}$. If $S(\Omega)$ exists, then there exist a defining function $\rho$ and $\eta_{2} \in(1, \infty)$ such that $\rho^{\eta_{2}}$ is strictly plurisubharmonic on $\bar{\Omega}^{\complement} \cap U$. Thus $\bar{\Omega}$ has a Stein neighborhood basis. In contrast to the Diederich-Fornæss index, the smoothness of the boundary does not imply the 
existence of $S(\Omega)$; worm domains provide an example ([6]). The author $([10])$ showed that the existence of Steinness index is equivalent to that of a strong Stein neighborhood basis. Moreover, the author found the following relation between two indices on worm domains $\Omega_{\beta}$;

$$
\frac{1}{D F\left(\Omega_{\beta}\right)}+\frac{1}{S\left(\Omega_{\beta}\right)}=2 .
$$

The purpose of this paper is to investigate whether those two indices are invariant under CR-diffeomorphisms. The author ([9]) proved that for relatively compact domains $\Omega_{1}$ and $\Omega_{2}$ in Stein manifolds with $C^{1}$-smooth boundary, if there exists CRdiffeomorphism $f: \partial \Omega_{1} \rightarrow \partial \Omega_{2}$, then $D F\left(\Omega_{1}\right)=D F\left(\Omega_{2}\right)$. The idea of the proof is the following: we first extend $f$ to a diffeomorphism $F: U_{1} \supset \bar{\Omega}_{1} \rightarrow U_{2} \supset \bar{\Omega}_{2}$ such that $\left.F\right|_{\Omega_{1}}: \Omega_{1} \rightarrow \Omega_{2}$ is a biholomorphism and $\left.F\right|_{\partial \Omega_{1}}=f$. Then for $0<\eta<1$ and a defining function $\rho$ of $\Omega_{2}$, if $-(-\rho)^{\eta}$ is strictly plurisubharmonic on $\Omega_{2}$, then $-(-(\rho \circ F))^{\eta}$ is also strictly plurisubharmonic on $\Omega_{1}$.

On the other hand, this approach for proving CR-invariance of the Steinness index does not work, because we can not extend CR-diffeomorphisms holomorphically outside the domain near the boundary. Instead, we characterize the Steinness index as well as the Diederich-Fornaess index on the set of weakly pseudoconvex boundary points in terms of a special 1-form, which we call D'Angelo 1-form (Definition 3.1).

Theorem 1.1. Let $\Omega \subset \subset \mathbb{C}^{n}$ be a pseudoconvex domain with $C^{k}(k \geq 3)$-smooth boundary. Let $\rho$ be a defining function of $\Omega$. Let $\alpha_{\rho}$ be a D'Angelo 1-form of $\rho$. Denote by $\omega_{\rho}:=\pi_{1,0} \alpha_{\rho}$ the projection of $\alpha_{\rho}$ onto its $(1,0)$-component. Then

$$
\begin{gathered}
D F(\Omega)=\sup _{\rho}\left\{0<\eta_{1}<1:\left(\frac{\eta_{1}}{1-\eta_{1}}\left(\omega_{\rho} \wedge \bar{\omega}_{\rho}\right)-\bar{\partial} \omega_{\rho}\right)(L, \bar{L}) \leq 0 \quad \forall p \in \Sigma, \quad \forall L \in \mathcal{N}_{p}\right\}, \\
S(\Omega)=\inf _{\rho}\left\{\eta_{2}>1:\left(\frac{\eta_{2}}{\eta_{2}-1}\left(\omega_{\rho} \wedge \bar{\omega}_{\rho}\right)+\bar{\partial} \omega_{\rho}\right)(L, \bar{L}) \leq 0 \quad \forall p \in \Sigma, \quad \forall L \in \mathcal{N}_{p}\right\},
\end{gathered}
$$

where $\Sigma$ is the set of weakly pseudoconvex boundary points and $\mathcal{N}_{p}$ is the null-space of Levi-form at $p$.

Then we prove that the two indices are invariant under CR-diffeomorphism by showing that D'Angelo 1-forms are invariant under CR-diffeomorphisms (Proposition 5.3).

Theorem 1.2. Let $\Omega_{1}$ and $\Omega_{2}$ be bounded domains in $\mathbb{C}^{n}$ with $C^{k}(k \geq 3)$-smooth boundaries. If $\partial \Omega_{1}$ and $\partial \Omega_{2}$ are $C R$-equivalent then

$$
D F\left(\Omega_{1}\right)=D F\left(\Omega_{2}\right) \quad \text { and } \quad S\left(\Omega_{1}\right)=S\left(\Omega_{2}\right)
$$

hold.

\section{Preliminaries}

We first fix the notation of this paper, unless otherwise mentioned.

- $\Omega$ : a bounded pseudoconvex domain with $C^{k}(k \geq 3)$-smooth boundary in $\mathbb{C}^{n}$. 
- $\rho$ : a defining function of $\Omega$.

- $\Sigma$ : the set of all weakly pseudoconvex points in $\partial \Omega$.

- $g$ : the standard Euclidean complex Hermitian metric in $\mathbb{C}^{n}$.

- $\nabla$ : the Levi-Civita connection of $g$.

- $\nabla \rho$ : the real gradient of $\rho$.

- $U$ : a tubular neighborhood of $\partial \Omega$.

- $\mathscr{L}_{\rho}$ : the Levi-form of $\rho$.

- $\mathcal{N}_{p}$ : the null-space of the Levi-form at $p \in \partial \Omega$.

Define

$$
N_{\rho}:=\frac{1}{\sqrt{\sum_{j=1}^{n}\left|\frac{\partial \rho}{\partial z_{j}}\right|^{2}}} \sum_{j=1}^{n} \frac{\partial \rho}{\partial \bar{z}_{j}} \frac{\partial}{\partial z_{j}}, \quad L_{n, \rho}:=\frac{1}{\sum_{j=1}^{n}\left|\frac{\partial \rho}{\partial z_{j}}\right|^{2}} \sum_{j=1}^{n} \frac{\partial \rho}{\partial \bar{z}_{j}} \frac{\partial}{\partial z_{j}} .
$$

We denote $N_{\rho}$ and $L_{n, \rho}$ by $N$ and $L_{n}$, respectively, if there is no ambiguity. Note that $N$ and $L_{n}$ depend on a defining function $\rho$, but $N$ is independent of $\rho$ on $\partial \Omega$. Also, note that $\operatorname{Re}\left\{N_{\rho}\right\}$ and $\operatorname{Re}\left\{L_{n}\right\}$ are real normal vector fields on $\partial \Omega$, and

$$
\|N\|=\sqrt{g(N, N)}=\frac{1}{\sqrt{2}}, \quad L_{n} \rho=1, \quad L_{n}=\frac{1}{\|\partial \rho\|} N=\frac{2}{\|\nabla \rho\|} N .
$$

Let $J$ be the complex structure of $\mathbb{C}^{n}$ and $T_{p}(\partial \Omega)$ be the real tangent space of $\partial \Omega$ at $p \in \partial \Omega$. Let $T_{p}^{c}(\partial \Omega):=J\left(T_{p}(\partial \Omega)\right) \cap T_{p}(\partial \Omega)$. Then the complexified tangent space of $T_{p}^{c}(\partial \Omega), \mathbb{C} T_{p}^{c}(\partial \Omega):=\mathbb{C} \otimes T_{p}^{c}(\partial \Omega)$, can be decomposed into the holomorphic tangent space $T_{p}^{1,0}(\partial \Omega)$ and the anti-holomorphic tangent space $T_{p}^{0,1}(\partial \Omega)$. We call $X$ a $(1,0)$ tangent vector if $X \in T_{p}^{1,0}(\partial \Omega)$.

Let $X, Y, Z$ be complex vector fields in $\mathbb{C}^{n}$. A direct calculation implies the following properties.

$$
\begin{gathered}
\mathscr{L}_{\rho}(X, Y)=g\left(\nabla_{X} \nabla \rho, Y\right)=X(\bar{Y} \rho)-\left(\nabla_{X} \bar{Y}\right) \rho, \\
Z g(X, Y)=g\left(\nabla_{Z} X, Y\right)+g\left(X, \nabla_{\bar{Z}} Y\right), \quad Z \rho=g(\nabla \rho, \bar{Z}), \\
\nabla_{X} \nabla_{Y}-\nabla_{Y} \nabla_{X}-\nabla_{[X, Y]}=0 .
\end{gathered}
$$

Let $d=\partial+\bar{\partial}$ be the exterior derivative and $d^{c}=i(\bar{\partial}-\partial)$.

Lemma 2.1. Let $\Omega \subset \subset \mathbb{C}^{n}$ be a pseudoconvex domain with $C^{k}(k \geq 2)$-smooth boundary, and $\rho$ be a defining function of $\Omega$. Suppose that $\mathscr{L}_{\rho}(L, L)(p)=0$ for $p \in \partial \Omega$ and $L \in T_{p}^{1,0}(\partial \Omega)$. Then $\mathscr{L}_{\rho}(L, T)(p)=0$ for all $T \in T_{p}^{1,0}(\partial \Omega)$.

Proof. Since $\Omega$ is pseudoconvex, the Levi-form of a defining function $\rho$ satisfies the Cauchy-Schwarz inequality on $T_{p}^{1,0}(\partial \Omega)$. Therefore, for all $T \in T_{p}^{1,0}(\partial \Omega)$,

$$
\left|\mathscr{L}_{\rho}(L, T)(p)\right|^{2} \leq\left|\mathscr{L}_{\rho}(L, L)(p)\right|\left|\mathscr{L}_{\rho}(T, T)(p)\right|=0,
$$

which completes the proof. 


\section{D'Angelo 1-form}

We first define a real 1 -form $\alpha$ which was first introduced by D'Angelo ([3], [4]). We call it D'Angelo 1-form. For a defining function $\rho$ of $\Omega$, define

$$
\eta_{\rho}=\frac{1}{2}(\partial \rho-\bar{\partial} \rho) \quad \text { and } \quad T_{\rho}=L_{n, \rho}-\bar{L}_{n, \rho}
$$

We denote $\eta_{\rho}$ and $T_{\rho}$ by $\eta$ and $T$, respectively, if there is no ambiguity. Then $\eta$ is a purely imaginary, non-vanishing 1 -form on $\partial \Omega$ that annihilates $T^{1,0}(\partial \Omega) \oplus T^{0,1}(\partial \Omega)$, and $T$ is a purely imaginary tangential vector field orthogonal to $T^{1,0}(\partial \Omega) \oplus T^{0,1}(\partial \Omega)$ such that $\eta(T) \equiv 1$.

Definition 3.1. A D'Angelo 1-form $\alpha_{\rho}$ on $\partial \Omega$ is defined by

$$
\alpha_{\rho}:=-\mathcal{L}_{T} \eta
$$

where $\mathcal{L}_{T}$ is the Lie derivative in the direction of $T$.

Remark 3.2. Note that the D'Angelo 1-form is defined from a defining function. On the other hand, we can also define the D'Angelo 1-form without using a defining function. Let $\eta$ is a purely imaginary, non-vanishing 1-form on $\partial \Omega$ that annihilates $T^{1,0}(\partial \Omega) \oplus$ $T^{0,1}(\partial \Omega)$, and $T_{\eta}$ is the (uniqe) purely imaginary tangential vector field orthogonal to $T^{1,0}(\partial \Omega) \oplus T^{0,1}(\partial \Omega)$ such that $\eta(T) \equiv 1$. Then the D'Angelo 1-form $\alpha_{\eta}$ of $\eta$ is defined by

$$
\alpha_{\eta}:=-\mathcal{L}_{T_{\eta}} \eta
$$

where $\mathcal{L}_{T_{\eta}}$ is the Lie derivative in the direction of $T_{\eta}$. We claim that those two definitions are equivalent. In order words, we prove that for any $\eta$ defined above there exists a defining function $\rho$ such that $\alpha_{\rho}=\alpha_{\eta}$. Fix a defining function $r$ of $\Omega$. Then since $\eta$ is non-vanishig, there exists a smooth function $\varphi$ on $\partial \Omega$ such that $\eta=e^{\varphi} \eta_{r}$ or $\eta=-e^{\varphi} \eta_{r}$. First, suppose that $\eta=e^{\varphi} \eta_{r}$. Then $T_{\eta}=e^{-\varphi} T_{r}$. Let $\rho:=e^{\varphi} r$. Then $\eta_{\rho}=e^{\varphi} \eta_{r}=\eta$ and $T_{\rho}=e^{-\varphi} T_{r}=T_{\eta}$. Therefore,

$$
\alpha_{\rho}=-\mathcal{L}_{T_{\rho}} \eta_{\rho}=-\mathcal{L}_{T_{\eta}} \eta=\alpha_{\eta} .
$$

If $\eta=-e^{\varphi} \eta_{r}$, then by the same argument above, $\alpha_{\rho}=-\mathcal{L}_{T_{\rho}} \eta_{\rho}=-\mathcal{L}_{\left(-T_{\eta}\right)}(-\eta)=\alpha_{\eta}$. In this paper, we use Definition 3.1 for our purpose.

We denote $\alpha_{\rho}$ by $\alpha$ if there is no ambiguity. Note that since $\eta$ and $T$ are purely imaginary, $\alpha$ is a real 1-form. Now we give known properties of $\alpha$ which we will use later. For more information about the D'Angelo 1-form, we refer the reader to [8].

Proposition $3.3([1])$. Let $\alpha$ be a D'Angelo 1-form on $\partial \Omega$. Then

$$
(d \alpha)_{p}(X, Y)=0
$$

for all $p \in \partial \Omega$ and $X, Y \in \mathcal{N}_{p} \oplus \overline{\mathcal{N}}_{p}$.

Lemma $3.4([1])$. Let $L_{1}, L$ be smooth $(1,0)$ tangent vector fields on $\partial \Omega$. Then

$$
\partial \rho\left(\left[L_{n}, \bar{L}\right]\right)=\mathscr{L}_{\rho}\left(L_{n}, L\right)
$$

and

$$
\partial \rho\left(\left[L_{1}, \bar{L}\right]\right)=\mathscr{L}_{\rho}\left(L_{1}, L\right)
$$


on $\partial \Omega$.

Proof. Let $L_{1}=\sum_{j=1}^{n} a_{j} \frac{\partial}{\partial z_{j}}$ and $L=\sum_{k=1}^{n} b_{k} \frac{\partial}{\partial z_{k}}$. Since $L_{n} \rho=1, L_{1} \rho=0$,

$$
\begin{gathered}
\sum_{j=1}^{n}\left(\frac{1}{\|\partial \rho\|^{2}} \frac{\partial \rho}{\partial \bar{z}_{j}}\right)\left(\frac{\partial \rho}{\partial z_{j}}\right)=1, \\
\sum_{j=1}^{n} a_{j} \frac{\partial \rho}{\partial z_{j}}=0
\end{gathered}
$$

imply

$$
\begin{gathered}
\sum_{j=1}^{n} \bar{L}\left(\frac{1}{\|\partial \rho\|^{2}} \frac{\partial \rho}{\partial \bar{z}_{j}}\right)\left(\frac{\partial \rho}{\partial z_{j}}\right)+\sum_{j=1}^{n}\left(\frac{1}{\|\partial \rho\|^{2}} \frac{\partial \rho}{\partial \bar{z}_{j}}\right) \bar{L}\left(\frac{\partial \rho}{\partial z_{j}}\right)=0 \\
\sum_{j=1}^{n} \bar{L}\left(a_{j}\right) \frac{\partial \rho}{\partial z_{j}}+\sum_{j=1}^{n} a_{j} \bar{L}\left(\frac{\partial \rho}{\partial z_{j}}\right)=0
\end{gathered}
$$

respectively. Therefore,

$$
\begin{aligned}
\partial \rho\left(\left[L_{n}, \bar{L}\right]\right) & =-\sum_{j=1}^{n} \bar{L}\left(\frac{1}{\|\partial \rho\|^{2}} \frac{\partial \rho}{\partial \bar{z}_{j}}\right) \frac{\partial \rho}{\partial z_{j}}=\sum_{j=1}^{n} \frac{1}{\|\partial \rho\|^{2}} \frac{\partial \rho}{\partial \bar{z}_{j}} \bar{L}\left(\frac{\partial \rho}{\partial z_{j}}\right) \\
& =\frac{1}{\|\partial \rho\|^{2}} \sum_{j=1, k=1}^{n} \frac{\partial^{2} \rho}{\partial z_{j} \partial \bar{z}_{k}} \frac{\partial \rho}{\partial \bar{z}_{j}} \bar{b}_{k}=\mathscr{L}_{\rho}\left(L_{n}, L\right),
\end{aligned}
$$

and

$$
\begin{aligned}
\partial \rho\left(\left[L_{1}, \bar{L}\right]\right) & =-\sum_{j=1}^{n} \bar{L}\left(a_{j}\right) \frac{\partial \rho}{\partial z_{j}}=\sum_{j=1}^{n} a_{j} \bar{L}\left(\frac{\partial \rho}{\partial z_{j}}\right) \\
& =\sum_{j=1, k=1}^{n} \frac{\partial^{2} \rho}{\partial z_{j} \partial \bar{z}_{k}} a_{j} \bar{b}_{k}=\mathscr{L}_{\rho}\left(L_{1}, L\right) .
\end{aligned}
$$

Lemma 3.5 (1]). Let $\alpha$ be a D'Angelo 1-form on $\partial \Omega$. Then

$$
\alpha(\bar{L})=\partial \rho\left(\left[L_{n}, \bar{L}\right]\right)=2 \frac{\mathscr{L}_{\rho}(N, L)}{\|\nabla \rho\|}
$$

on $\partial \Omega$ for a $(1,0)$ vector field $L$ on $\partial \Omega$.

Proof. Lemma 3.4 implies that

$$
\begin{aligned}
\alpha(\bar{L}) & =-\left(\mathcal{L}_{T} \eta\right)(\bar{L})=-T(\eta(\bar{L}))+\eta([T, \bar{L}])=\eta([T, \bar{L}])=\frac{1}{2}(\partial \rho-\bar{\partial} \rho)([T, \bar{L}]) \\
& =\partial \rho\left(\left[L_{n}-\bar{L}_{n}, \bar{L}\right]\right)=\partial \rho\left(\left[L_{n}, \bar{L}\right]\right)=\mathscr{L}_{\rho}\left(L_{n}, L\right)=2 \frac{\mathscr{L}_{\rho}(N, L)}{\|\nabla \rho\|} .
\end{aligned}
$$

Here, we used $(\partial \rho+\bar{\partial} \rho)([T, \bar{L}])=d \rho([T, \bar{L}])=0$ (because $[T, \bar{L}]$ is tangential), and $\partial \rho\left(\left[\bar{L}_{n}, \bar{L}\right]\right)=0$ (because $\left[\bar{L}_{n}, \bar{L}\right]$ is of type $\left.(0,1)\right)$. 


\section{Characterization of two indices in terms of D'Angelo 1-form}

The author ([10]), exploiting the idea of [7], characterized the Steinness index by way of a differential inequality on the set of weakly pseudoconvex boundary points (in fact, on the set of infinite type boundary points in the sense of D'Angelo). Also, we may induce a similar description for the Diederich-Fornaess index by the same argument. The two descriptions are the following.

Throughout this section, let $L$ be an arbitrary $(1,0)$ vector field defined on a neighborhood $U \supset \partial \Omega$ with $L \rho=0$ and define

$$
\Sigma_{L}:=\left\{p \in \partial \Omega: \mathscr{L}_{\rho}(L, L)(p)=0\right\} .
$$

Theorem 4.1.

$$
D F(\Omega)=\sup _{\rho}\left\{0<\eta_{1}<1: \frac{1}{1-\eta_{1}} \frac{\left|\mathscr{L}_{\rho}(L, N)\right|^{2}}{\|\nabla \rho\|^{2}}+\frac{1}{2} \frac{N \mathscr{L}_{\rho}(L, L)}{\|\nabla \rho\|} \leq 0 \text { on } \Sigma_{L} \forall L\right\},
$$

where the supremum is taken over all smooth defining functions $\rho$.

Theorem 4.2 (Yum [10]).

$$
S(\Omega)=\inf _{\rho}\left\{\eta_{2}>1: \frac{1}{\eta_{2}-1} \frac{\left|\mathscr{L}_{\rho}(L, N)\right|^{2}}{\|\nabla \rho\|^{2}}-\frac{1}{2} \frac{N \mathscr{L}_{\rho}(L, L)}{\|\nabla \rho\|} \leq 0 \text { on } \Sigma_{L} \forall L\right\},
$$

where the infimum is taken over all smooth defining functions $\rho$.

Remark 4.3. We give here an explaination about the difference of Theorem 4.1 and the formula of Liu (Thoerem 2.9 in [7]). Liu dealt with a defining function of the form $r e^{\psi}$, where $r$ is a defining function and $\psi$ is a smooth function near the boundary. If we apply the same argument as Liu for a defining function $\rho$, then one can conclude Theorem 4.1. If we replace $\rho$ by $r e^{\psi}$ in Theorem 4.1 (using Lemma 2.2 in [10]), one gets a formula which is equivalent to the formula of Liu.

The two theorems above imply that the Diederich-Fornaess index and Steinness index are completely determined by the two values $\frac{\left|\mathscr{L}_{\rho}(L, N)\right|^{2}}{\|\nabla \rho\|^{2}}$ and $\frac{N \mathscr{L}_{\rho}(L, L)}{\|\nabla \rho\|}$ on the set of weakly pseudoconvex boundary points. From Lemma 3.5, we know that

$$
\alpha(\bar{L})=2 \frac{\mathscr{L}_{\rho}(N, L)}{\|\nabla \rho\|}
$$

for a D'Angelo 1-form $\alpha$. We now describe the second value in terms of $\alpha$.

Lemma 4.4. Let $\alpha$ be a smooth real 1-form on $\mathbb{C}^{n}$. Let $X \in T^{1,0}\left(\mathbb{C}^{n}\right)$ and $\bar{Y} \in T^{0,1}\left(\mathbb{C}^{n}\right)$. Then the following equations hold.

$$
\begin{aligned}
(d \alpha)(X, \bar{Y}) & =X(\alpha(\bar{Y}))-\bar{Y}(\alpha(X))-\alpha\left(\nabla_{X} \bar{Y}\right)+\alpha\left(\nabla_{\bar{Y}} X\right), \\
\left(d^{c} \alpha\right)(X, \bar{Y}) & =i\left[-X(\alpha(\bar{Y}))-\bar{Y}(\alpha(X))+\alpha\left(\nabla_{X} \bar{Y}\right)+\alpha\left(\nabla_{\bar{Y}} X\right)\right] .
\end{aligned}
$$

Proof. The first equation follows by the definition of exterior derivative :

$$
\begin{aligned}
(d \alpha)(X, \bar{Y}) & =X(\alpha(\bar{Y}))-\bar{Y}(\alpha(X))-\alpha([X, \bar{Y}]) \\
& =X(\alpha(\bar{Y}))-\bar{Y}(\alpha(X))-\alpha\left(\nabla_{X} \bar{Y}\right)+\alpha\left(\nabla_{\bar{Y}} X\right) .
\end{aligned}
$$


Since $\alpha$ is real, we may write

$$
\alpha=\sum_{l=1}^{n}\left(a_{l} d z_{l}+\bar{a}_{l} d \bar{z}_{l}\right), \quad X=\sum_{j=1}^{n} x_{j} \frac{\partial}{\partial z_{j}}, \quad Y=\sum_{k=1}^{n} \bar{y}_{k} \frac{\partial}{\partial \bar{z}_{k}}
$$

for some $a_{j} \in \mathbb{C}$. Then

$$
\begin{gathered}
d^{c} \alpha=i(\bar{\partial}-\partial) \alpha \\
=i \sum_{l, m=1}^{n}\left[\left(\frac{\partial a_{l}}{\partial \bar{z}_{m}} d \bar{z}_{m} \wedge d z_{l}+\frac{\partial \bar{a}_{l}}{\partial \bar{z}_{m}} d \bar{z}_{m} \wedge d \bar{z}_{l}\right)-\left(\frac{\partial a_{l}}{\partial z_{m}} d z_{m} \wedge d z_{l}+\frac{\partial \bar{a}_{l}}{\partial z_{m}} d z_{m} \wedge d \bar{z}_{l}\right)\right] \\
\left(d^{c} \alpha\right)(X, \bar{Y})=i \sum_{j, k=1}^{n}\left[-\frac{\partial a_{j}}{\partial \bar{z}_{k}} x_{j} \bar{y}_{k}-\frac{\partial \bar{a}_{k}}{\partial z_{j}} x_{j} \bar{y}_{k}\right] \\
X(\alpha(\bar{Y}))=X\left(\sum_{l=1}^{n} \bar{a}_{l} \bar{y}_{l}\right)=\sum_{j, k=1}^{n} x_{j} \frac{\partial \bar{a}_{k}}{\partial z_{j}} \bar{y}_{k}+\sum_{j, k=1}^{n} x_{j} \bar{a}_{k} \frac{\partial \bar{y}_{k}}{\partial z_{j}} \\
\bar{Y}(\alpha(X))=\bar{Y}\left(\sum_{l=1}^{n} a_{l} x_{l}\right)=\sum_{j, k=1}^{n} \bar{y}_{k} \frac{\partial a_{j}}{\partial \bar{z}_{k}} x_{j}+\sum_{j, k=1}^{n} \bar{y}_{k} a_{j} \frac{\partial x_{j}}{\partial \bar{z}_{k}} \\
\nabla_{X} \bar{Y}=\sum_{j, k=1}^{n} x_{j} \frac{\partial \bar{y}_{k}}{\partial z_{j}} \frac{\partial}{\partial \bar{z}_{k}}, \quad \nabla_{\bar{Y}} X=\sum_{j, k=1}^{n} \bar{y}_{k} \frac{\partial x_{j}}{\partial \bar{z}_{k}} \frac{\partial}{\partial z_{j}}, \\
\alpha\left(\nabla_{X} \bar{Y}\right)=\sum_{j, k=1}^{n} \bar{a}_{k} x_{j} \frac{\partial \bar{y}_{k}}{\partial z_{j}}, \quad \alpha\left(\nabla \bar{Y}_{Y} X\right)=\sum_{j, k=1}^{n} a_{j} \bar{y}_{k} \frac{\partial x_{j}}{\partial \bar{z}_{k}},
\end{gathered}
$$

Therefore,

$$
\left(d^{c} \alpha\right)(X, \bar{Y})=i\left[-X(\alpha(\bar{Y}))-\bar{Y}(\alpha(X))+\alpha\left(\nabla_{X} \bar{Y}\right)+\alpha\left(\nabla_{\bar{Y}} X\right)\right] .
$$

\section{Lemma 4.5.}

$$
\frac{\left|\mathscr{L}_{\rho}(L, N)\right|^{2}}{\|\nabla \rho\|^{2}}+\frac{1}{2} \frac{N \mathscr{L}_{\rho}(L, L)}{\|\nabla \rho\|}=\frac{1}{4} g\left(\nabla_{L} \nabla_{L_{n}} \nabla \rho, L\right)
$$

holds at $p \in \Sigma_{L}$ for all $L$.

Proof. Fix $p \in \Sigma_{L}$. Then

$$
\begin{aligned}
\frac{1}{2} \frac{N \mathscr{L}_{\rho}(L, L)}{\|\nabla \rho\|} & =\frac{1}{4} L_{n} \mathscr{L}_{\rho}(L, L)=\frac{1}{4} L_{n} g\left(\nabla_{L} \nabla \rho, L\right) \\
& =\frac{1}{4} g\left(\nabla_{L_{n}} \nabla_{L} \nabla \rho, L\right)+\frac{1}{4} g\left(\nabla_{L} \nabla \rho, \nabla_{\bar{L}_{n}} L\right) \\
& =\frac{1}{4} g\left(\nabla_{L} \nabla_{L_{n}} \nabla \rho, L\right)+\frac{1}{4} g\left(\nabla_{\left[L_{n}, L\right]} \nabla \rho, L\right)+\frac{1}{4} g\left(\nabla_{L} \nabla \rho, \nabla_{\bar{L}_{n}} L\right)
\end{aligned}
$$


at $p$. If $L$ vanishes at $p$, then the last three terms are all zero and hence $N \mathscr{L}_{\rho}(L, L)=0$. Thus the equation 4.1 holds. Therefore, we assume that $L \neq 0$ at $p$.

Let $\left\{\sqrt{2} T_{1}, \cdots, \sqrt{2} T_{n-2}, \sqrt{2} T_{n-1}\right\}$ be an orthonormal basis of $T_{p}^{1,0}(\partial \Omega)$ with $\sqrt{2} T_{n-1}=$ $\frac{L}{\|L\|}$. Then first,

$$
\begin{aligned}
& g\left(\nabla_{L} \nabla \rho, \nabla_{\bar{L}_{n}} L\right) \\
= & g\left(\nabla_{L} \nabla \rho, \sum_{j=1}^{n-1} g\left(\nabla_{\bar{L}_{n}} L, \sqrt{2} T_{j}\right) \sqrt{2} T_{j}+g\left(\nabla_{\bar{L}_{n}} L, \sqrt{2} N\right) \sqrt{2} N\right) \\
= & \sum_{j=1}^{n-1} \overline{2 g\left(\nabla_{\bar{L}_{n}} L, T_{j}\right)} \mathscr{L}_{\rho}\left(L, T_{j}\right)+2 \overline{g\left(\nabla_{\bar{L}_{n}} L, N\right)} \mathscr{L}_{\rho}(L, N) \\
= & 2 \overline{g\left(\nabla_{\bar{L}_{n}} L, N\right)} \mathscr{L}_{\rho}(L, N) \\
= & 4 \overline{g\left(\nabla_{\bar{N}} L, N\right)} \frac{\mathscr{L}_{\rho}(L, N)}{\|\nabla \rho\|} .
\end{aligned}
$$

Here, we used Lemma 2.1 in the third equality above.

$$
\begin{aligned}
& \mathscr{L}_{\rho}(N, L)=N(\bar{L} \rho)-\left(\nabla_{N} \bar{L}\right) \rho=-\left(\nabla_{N} \bar{L}\right) \rho \\
& \Rightarrow \quad-\mathscr{L}_{\rho}(L, N)=\left(\nabla_{\bar{N}} L\right) \rho=2 g\left(\nabla_{\bar{N}} L, N\right)(N \rho)=g\left(\nabla_{\bar{N}} L, N\right)\|\nabla \rho\| \\
& \Rightarrow \quad g\left(\nabla_{\bar{N}} L, N\right)=-\frac{\mathscr{L}_{\rho}(L, N)}{\|\nabla \rho\|} .
\end{aligned}
$$

Therefore,

$$
g\left(\nabla_{L} \nabla \rho, \nabla_{\bar{L}_{n}} L\right)=-4 \frac{\left|\mathscr{L}_{\rho}(L, N)\right|^{2}}{\|\nabla \rho\|^{2}}
$$

Second, by the same argument as above,

$$
\begin{gathered}
g\left(\nabla_{\left[L_{n}, L\right]} \nabla \rho, L\right)=\overline{g\left(\nabla_{L} \nabla \rho,\left[L_{n}, L\right]\right)}=2 g\left(\left[L_{n}, L\right], N\right) \overline{\mathscr{L}_{\rho}(L, N)} . \\
g\left(\left[L_{n}, L\right], N\right)=g\left(\left[L_{n}, L\right], N+\bar{N}\right)=\frac{1}{\|\nabla \rho\|} g\left(\left[L_{n}, L\right], \nabla \rho\right)=\frac{1}{\|\nabla \rho\|}\left[L_{n}, L\right] \rho \\
=\frac{1}{\|\nabla \rho\|}\left(L_{n}(L \rho)-L\left(L_{n} \rho\right)\right)=0 .
\end{gathered}
$$

The last equation follows from $L \rho=0$ and $L_{n} \rho=1$. Therefore,

$$
g\left(\nabla_{\left[L_{n}, L\right]} \nabla \rho, L\right)=0 .
$$

All together, we have

$$
\frac{\left|\mathscr{L}_{\rho}(L, N)\right|^{2}}{\|\nabla \rho\|^{2}}+\frac{1}{2} \frac{N \mathscr{L}_{\rho}(L, L)}{\|\nabla \rho\|}=\frac{1}{4} g\left(\nabla_{L} \nabla_{L_{n}} \nabla \rho, L\right) .
$$


Proposition 4.6. Let $\alpha$ be a D'Angelo 1-form. Then

$$
\frac{\left|\mathscr{L}_{\rho}(L, N)\right|^{2}}{\|\nabla \rho\|^{2}}+\frac{1}{2} \frac{N \mathscr{L}_{\rho}(L, L)}{\|\nabla \rho\|}=\frac{i}{8}\left(d^{c} \alpha\right)(L, \bar{L})
$$

holds at $p \in \Sigma_{L}$ for all $L$.

Proof. By Lemma 4.5,

$$
\begin{aligned}
& \frac{\left|\mathscr{L}_{\rho}(L, N)\right|^{2}}{\|\nabla \rho\|^{2}}+\frac{1}{2} \frac{N \mathscr{L}_{\rho}(L, L)}{\|\nabla \rho\|} \\
= & \frac{1}{4} g\left(\nabla_{L} \nabla_{L_{n}} \nabla \rho, L\right) \\
= & \frac{1}{4} L g\left(\nabla_{L_{n}} \nabla \rho, L\right)-\frac{1}{4} g\left(\nabla_{L_{n}} \nabla \rho, \nabla_{\bar{L}} L\right) \\
= & \frac{1}{4} L \mathscr{L}_{\rho}\left(L_{n}, L\right)-\frac{1}{4} \mathscr{L}_{\rho}\left(L_{n}, \nabla_{\bar{L}} L\right)
\end{aligned}
$$

Since $\mathscr{L}_{\rho}(L, L)=0$ at $p$,

$$
0=\mathscr{L}_{\rho}(L, L)=\bar{L}(L \rho)-\left(\nabla_{\bar{L}} L\right) \rho=-\left(\nabla_{\bar{L}} L\right) \rho
$$

implies that $\left(\nabla_{\bar{L}} L\right) \in T_{p}^{1,0}(\partial \Omega)$. Then by Lemma 3.5,

$$
\begin{aligned}
\alpha(\bar{L}) & =2 \frac{\mathscr{L}_{\rho}(N, L)}{\|\nabla \rho\|}=\mathscr{L}_{\rho}\left(L_{n}, L\right), \\
\alpha\left(\nabla_{L} \bar{L}\right) & =2 \frac{\mathscr{L}_{\rho}\left(N, \nabla_{\bar{L}} L\right)}{\|\nabla \rho\|}=\mathscr{L}_{\rho}\left(L_{n}, \nabla_{\bar{L}} L\right) .
\end{aligned}
$$

Note that we did not use $\mathscr{L}_{\rho}(L, L)=0$ in the proof of Lemma 3.5, we just used $L \rho=0$. Altogether, we have

$$
\frac{\left|\mathscr{L}_{\rho}(L, N)\right|^{2}}{\|\nabla \rho\|^{2}}+\frac{1}{2} \frac{N \mathscr{L}_{\rho}(L, L)}{\|\nabla \rho\|}=\frac{1}{4} L(\alpha(\bar{L}))-\frac{1}{4} \alpha\left(\nabla_{L} \bar{L}\right) .
$$

Now by Lemma 4.4 and the above equation,

$$
\frac{1}{8}\left(d \alpha+i d^{c} \alpha\right)(L, \bar{L})=\frac{1}{4} L(\alpha(\bar{L}))-\frac{1}{4} \alpha\left(\nabla_{L} \bar{L}\right)=\frac{\left|\mathscr{L}_{\rho}(L, N)\right|^{2}}{\|\nabla \rho\|^{2}}+\frac{1}{2} \frac{N \mathscr{L}_{\rho}(L, L)}{\|\nabla \rho\|}
$$

Since $d \alpha(L, \bar{L})=0$ by Proposition 3.3 , we have

$$
\frac{\left|\mathscr{L}_{\rho}(L, N)\right|^{2}}{\|\nabla \rho\|^{2}}+\frac{1}{2} \frac{N \mathscr{L}_{\rho}(L, L)}{\|\nabla \rho\|}=\frac{i}{8}\left(d^{c} \alpha\right)(L, \bar{L}) .
$$

Proof of Theorem 1.1. We prove it for the Steinness index case. Since $\alpha$ is a real 1 -form, we may write it as $\alpha=\omega+\bar{\omega}$. Then

$$
\frac{i}{2} d^{c} \alpha(L, \bar{L})=\frac{1}{2}\left(d+i d^{c}\right) \alpha(L, \bar{L})=\partial \alpha(L, \bar{L})=\partial \bar{\omega}(L, \bar{L})=-\bar{\partial} \omega(L, \bar{L}) .
$$


Here, Proposition 3.3 is used in the first equality. Hence, from Lemma 3.5 and Proposition 4.6.

$$
\begin{aligned}
& \frac{1}{\eta_{2}-1} \frac{\left|\mathscr{L}_{\rho}(L, N)\right|^{2}}{\|\nabla \rho\|^{2}}-\frac{1}{2} \frac{N \mathscr{L}_{\rho}(L, L)}{\|\nabla \rho\|} \leq 0 \\
\Leftrightarrow & \left(\frac{\eta_{2}}{\eta_{2}-1}(\omega \wedge \bar{\omega})-\frac{i}{2} d^{c} \alpha\right)(L, \bar{L}) \leq 0 \\
\Leftrightarrow & \left(\frac{\eta_{2}}{\eta_{2}-1}(\omega \wedge \bar{\omega})+\bar{\partial} \omega\right)(L, \bar{L}) \leq 0 .
\end{aligned}
$$

Therefore, the theorem is proved by Theorem 4.2. For the Diederich-Fornaess index case, it follows from the same argument as above.

Remark 4.7. We give an alternative proof for Lemma 2.2 in [10]. Let $\widetilde{\rho}$ and $\rho$ be $C^{k}(k \geq 3)$-smooth defining functions of $\Omega$. Then there exists a $C^{k-1}$-smooth realvalued function $\psi$ such that $\widetilde{\rho}=\rho e^{\psi}$. Let $\widetilde{\eta}=\eta_{\widetilde{\rho}}, \eta=\eta_{\rho}, \widetilde{T}=T_{\widetilde{\rho}}$ and $T=T_{\rho}$ as in Section 3. Let $\widetilde{\alpha}=-\mathcal{L}_{\widetilde{T}} \widetilde{\eta}$ and $\alpha=-\mathcal{L}_{T} \eta$. By a direct calculation, $\widetilde{\eta}=e^{\psi} \eta$ and $\widetilde{T}=e^{-\psi} T$. Then for $X \in T^{1,0}(\partial \Omega)$, as in the proof of Lemma 3.5,

$$
\widetilde{\alpha}(X)=\widetilde{\eta}([\widetilde{T}, X])=e^{\psi} \eta\left(\left[e^{-\psi} T, X\right]\right)=\eta([T, X])+X \psi=\alpha(X)+d \psi(X) .
$$

Since $\widetilde{\alpha}, \alpha, \psi$ are real,

$$
\widetilde{\alpha}(\bar{X})=\alpha(\bar{X})+d \psi(\bar{X}) .
$$

Moreover, by the equation (4.4) and the above equation, for $L \in \mathcal{N}_{p}, p \in \partial \Omega$,

$$
\begin{aligned}
i d^{c} \widetilde{\alpha}(L, \bar{L}) & =2 L(\widetilde{\alpha}(\bar{L}))-2 \widetilde{\alpha}\left(\nabla_{L} \bar{L}\right) \\
& =2 L(\alpha(\bar{L})+\bar{L} \psi)-2\left(\alpha\left(\nabla_{L} \bar{L}\right)+\left(\nabla_{L} \bar{L}\right) \psi\right) \\
& =2 L(\alpha(\bar{L}))-2 \alpha\left(\nabla_{L} \bar{L}\right)+2 L(\bar{L} \psi)-2\left(\nabla_{L} \bar{L}\right) \psi \\
& =i d^{c} \alpha(L, \bar{L})-i d d^{c} \psi(L, \bar{L}) .
\end{aligned}
$$

In the second equlity, we used $\nabla_{L} \bar{L} \in T^{0,1}(\partial \Omega)$ (see the equation (4.3)).

\section{CR-invariance of two indices}

In the previous section, we showed that the Diederich-Fornaess index and Steinness index are completely determined by D'Angelo 1-forms on the set of weakly pseudoconvex boundary points. In this section, we prove that those two indices are invariant under CR-diffeomorphisms by showing that D'Angelo 1-forms are invariant under CRdiffeomorphisms in some sense.

Definition 5.1. Let $\Omega_{1}$ and $\Omega_{2}$ be domains in $\mathbb{C}^{n}(n \geq 2)$ with $C^{k}(k \geq 1)$-smooth boundaries. A $C^{k}$-smooth function $f: \Omega_{1} \rightarrow \mathbb{C}$ is called a $C R$-function if

$$
\bar{L} f=0 \text {, }
$$


for all $p \in \partial \Omega_{1}$ and $L \in T_{p}^{1,0}\left(\partial \Omega_{1}\right)$. A $C^{k}$-smooth function $F: \partial \Omega_{1} \rightarrow \partial \Omega_{2}$ is called a $C R$-map if

$$
F\left(T_{p}^{1,0}\left(\partial \Omega_{1}\right)\right) \subset T_{f(p)}^{1,0}\left(\partial \Omega_{2}\right)
$$

for all $p \in \partial \Omega_{1}$. A $C^{k}$-smooth function $F: \partial \Omega_{1} \rightarrow \partial \Omega_{2}$ is called a $C R$-diffeomorphism if it is a CR-map and a diffeomorphism.

Theorem 5.2 (Boggess [2]). Suppose $M$ is a $C^{k}, k \geq 2$, generic CR submanifold of $\mathbb{C}^{n}$ with real dimension $2 n-d, 1 \leq d \leq n$. If $f$ is a $C^{k} C R$-function on $M$, then there exists a $C^{k}$ function $F$ defined on $\mathbb{C}^{n}$ such that $\bar{\partial} F$ vanishes on $M$ to order $k-1$ and $\left.F\right|_{M}=f$.

Proposition 5.3. Let $\Omega_{1}$ and $\Omega_{2}$ be bounded pseudoconvex domains in $\mathbb{C}^{n}$ with $C^{k}(k \geq$ 3)-smooth boundaries, and $\rho$ be a defining function of $\Omega_{2}$. Suppose that there exists a $C^{k}$-smooth CR-diffeomorphism $f: \partial \Omega_{1} \rightarrow \partial \Omega_{2}$. Then

$$
\begin{aligned}
\alpha_{(\rho \circ f)}(\bar{L})(p) & =\alpha_{\rho}\left(\overline{f_{*}(L)}\right)(f(p)), \\
d^{c} \alpha_{(\rho \circ f)}(L, \bar{L})(p) & =d^{c} \alpha_{\rho}\left(f_{*}(L), \overline{f_{*}(L)}\right)(f(p)),
\end{aligned}
$$

for all $p \in \partial \Omega_{1}$ and $L \in \mathcal{N}_{p}$.

Proof. Let $\left\{z_{j}\right\}_{j=1}^{n}$ and $\left\{w_{j}\right\}_{j=1}^{n}$ be coordinates of $\Omega_{1} \subset \mathbb{C}^{n}$ and $\Omega_{2} \subset \mathbb{C}^{n}$, respectively. By Theorem 5.2, we may extend $f$ to $F$ such that $\bar{\partial} F$ vanishes on $\partial \Omega_{1}$ to order $k-1$ and $\left.F\right|_{M}=f$. We will denote the extension $F$ again by $f$. Then,

$$
\mathscr{L}_{(\rho \circ f)}(X, Y)(p)=\mathscr{L}_{\rho}\left(f_{*}(X), f_{*}(Y)\right)(f(p))
$$

for all $p \in \partial \Omega_{1}$ and $X, Y \in T_{p}^{1,0}\left(\partial \Omega_{1}\right)$. Denote

$$
L_{n, \rho}:=\frac{1}{\sum_{j=1}^{n}\left|\frac{\partial \rho}{\partial w_{j}}\right|^{2}} \sum_{j=1}^{n} \frac{\partial \rho}{\partial \bar{w}_{j}} \frac{\partial}{\partial w_{j}} .
$$

Claim. $f_{*}\left(L_{n,(\rho \circ f)}\right)=L_{n, \rho}+\widetilde{L}$ for some $\widetilde{L} \in T_{f(p)}^{1,0}\left(\partial \Omega_{2}\right)$.

Let $f=\left(f_{1}, \cdots, f_{n}\right)$. Then for $p \in \partial \Omega_{1}$ and $q:=f(p) \in \partial \Omega_{2}$,

$$
\begin{gathered}
\left.\frac{\partial(\rho \circ f)}{\partial \bar{z}_{j}}\right|_{p}=\left.\left.\sum_{k=1}^{n} \frac{\partial \rho}{\partial \bar{w}_{k}}\right|_{q} \frac{\partial \bar{f}_{k}}{\partial \bar{z}_{j}}\right|_{p},\left.\quad \frac{\partial(\rho \circ f)}{\partial z_{j}}\right|_{p}=\left.\left.\sum_{k=1}^{n} \frac{\partial \rho}{\partial w_{k}}\right|_{q} \frac{\partial f_{k}}{\partial z_{j}}\right|_{p}, \\
\left.\left|\frac{\partial(\rho \circ f)}{\partial z_{j}}\right|_{p}\right|_{k, l=1} ^{n}=\left.\left.\left.\left.\sum_{\frac{\partial \rho}{\bar{w}_{k}}}\right|_{q} \frac{\partial \bar{f}_{k}}{\partial \bar{z}_{j}}\right|_{p} \frac{\partial \rho}{\partial w_{l}}\right|_{q} \frac{\partial f_{l}}{\partial z_{j}}\right|_{p},
\end{gathered}
$$

and

$$
f_{*}\left(\left.\left.\sum_{j=1}^{n} \frac{\partial(\rho \circ f)}{\partial \bar{z}_{j}}\right|_{p} \frac{\partial}{\partial z_{j}}\right|_{p}\right)=\left.\left.\left.\left.\sum_{j, k, l=1}^{n} \frac{\partial f_{l}}{\partial z_{j}}\right|_{p} \frac{\partial \rho}{\partial \bar{w}_{k}}\right|_{q} \frac{\partial \bar{f}_{k}}{\partial \bar{z}_{j}}\right|_{p} \frac{\partial}{\partial w_{l}}\right|_{q} .
$$

Hence, $f_{*}\left(L_{n,(\rho \circ f)}\right) \rho \equiv 1$ on $\partial \Omega_{2}$. Let $f_{*}\left(L_{n,(\rho \circ f)}\right)=a L_{n, \rho}+\widetilde{L}$ for some $a \in \mathbb{C}$ and $\widetilde{L} \in T_{f(p)}^{1,0}\left(\partial \Omega_{2}\right)$. Then

$$
1=f_{*}\left(L_{n,(\rho \circ f)}\right) \rho=a L_{n, \rho} \rho+\widetilde{L} \rho=a
$$


and the claim is proved.

Now, by Lemma 3.5 and the claim above,

$$
\begin{aligned}
\alpha_{(\rho \circ f)}(\bar{L})(p) & =\mathscr{L}_{(\rho \circ f)}\left(L_{n,(\rho \circ f)}, L\right)(p) \\
& =\mathscr{L}_{\rho}\left(f_{*}\left(L_{n,(\rho \circ f)}\right), f_{*}(L)\right)(f(p)) \\
& =\mathscr{L}_{\rho}\left(L_{n, \rho}, f_{*}(L)\right)(f(p))+\mathscr{L}_{\rho}\left(\widetilde{L}, f_{*}(L)\right)(f(p)) \\
& =\alpha_{\rho}\left(\overline{f_{*}(L)}\right)(f(p)) .
\end{aligned}
$$

The explanation for the fourth equality is the following. Since $f$ is a CR-map and $L \in \mathcal{N}_{p}$, we have $f_{*}(L) \in \mathcal{N}_{f(p)}$. Hence by Lemma 2.1, $\mathscr{L}_{\rho}\left(\widetilde{L}, f_{*}(L)\right)(f(p))=0$. Therefore, the first equation (5.1) is proved.

For the second equation, we first claim that $\overline{f_{*}\left(\nabla_{\bar{L}} L\right)}=\nabla_{f_{*}(L)} \overline{f_{*}(L)}$ on $\partial \Omega_{2}$. Let $f^{-1}=\left(f_{1}^{-1}, \cdots, f_{n}^{-1}\right)$ and

$$
L=\sum_{j=1}^{n} a_{j} \frac{\partial}{\partial z_{j}}
$$

Then

$$
\begin{array}{r}
\nabla_{\bar{L}} L=\sum_{j, k=1}^{n} \bar{a}_{k} \frac{\partial a_{j}}{\partial \bar{z}_{k}} \frac{\partial}{\partial z_{j}}, \quad \overline{f_{*}\left(\nabla_{\bar{L}} L\right)}=\sum_{j, k, l=1}^{n} a_{k} \frac{\partial \bar{a}_{j}}{\partial z_{k}} \frac{\partial \bar{f}_{l}}{\partial \bar{z}_{j}} \frac{\partial}{\partial \bar{w}_{l}} \\
f_{*}(L)=\sum_{j, k=1}^{n} a_{j} \frac{\partial f_{k}}{\partial z_{j}} \frac{\partial}{\partial w_{k}}, \quad \overline{f_{*}(L)}=\sum_{i, l=1}^{n} \bar{a}_{i} \frac{\partial \bar{f}_{l}}{\partial \bar{z}_{i}} \frac{\partial}{\partial \bar{w}_{l}}
\end{array}
$$

Therefore,

$$
\begin{aligned}
\nabla_{f_{*}(L)} \overline{f_{*}(L)} & =\sum_{i, j, k, l, m=1}^{n} a_{j} \frac{\partial f_{k}}{\partial z_{j}} \frac{\partial \bar{a}_{i}}{\partial z_{m}} \frac{\partial f_{m}^{-1}}{\partial w_{k}} \frac{\partial \bar{f}_{l}}{\partial \bar{z}_{i}} \frac{\partial}{\partial \bar{w}_{l}}=\sum_{i, j, l, m=1}^{n} a_{j} \frac{\partial \bar{a}_{i}}{\partial z_{m}} \delta_{j}^{m} \frac{\partial \bar{f}_{l}}{\partial \bar{z}_{i}} \frac{\partial}{\partial \bar{w}_{l}} \\
& =\sum_{i, j, l=1}^{n} a_{j} \frac{\partial \bar{a}_{i}}{\partial z_{j}} \frac{\partial \bar{f}_{l}}{\partial \bar{z}_{i}} \frac{\partial}{\partial \bar{w}_{l}}=\overline{f_{*}\left(\nabla_{\bar{L}} L\right)},
\end{aligned}
$$

where $\delta_{j}^{m}$ is the Kronecker delta.

Now by Lemma 4.4.

$$
\begin{aligned}
i\left(d^{c} \alpha_{(f \circ \rho)}\right)(L, \bar{L})(p) & =\left(d \alpha_{(f \circ \rho)}+i d^{c} \alpha_{(f \circ \rho)}\right)(L, \bar{L})(p) \\
& =2 L\left(\alpha_{(f \circ \rho)}(\bar{L})\right)(p)-2 \alpha_{(f \circ \rho)}\left(\nabla_{L} \bar{L}\right)(p) \\
& =2 L\left(\alpha_{\rho}\left(\overline{f_{*}(L)}\right) \circ f\right)(p)-2 \alpha_{\rho}\left(\overline{f_{*}\left(\nabla_{\bar{L}} L\right)}\right)(f(p)) \\
& =2 f_{*}(L)\left(\alpha_{\rho}\left(\overline{f_{*}(L)}\right)\right)(f(p))-2 \alpha_{\rho}\left(\nabla_{f_{*}(L)} \overline{f_{*}(L)}\right)(f(p)) \\
& =i d^{c} \alpha_{\rho}\left(f_{*}(L), \overline{f_{*}(L)}\right)(f(p)) .
\end{aligned}
$$


Proof of Theorem 1.2. We prove that $S\left(\Omega_{1}\right)=S\left(\Omega_{2}\right)$. If $S\left(\Omega_{1}\right)$ and $S\left(\Omega_{2}\right)$ are both infinity, then we are done. Hence, we may assume $S\left(\Omega_{1}\right)<\infty$. Then, since $\Omega_{1}$ admits a Stein neighborhood basis, $\Omega_{1}$ is a pseudoconvex domain. Moreover, CR-equivalance of $\partial \Omega_{1}$ and $\partial \Omega_{2}$ implies that $\Omega_{2}$ is a pseudoconvex domain. Therefore, we can apply Theorem 1.1 for both $\Omega_{1}$ and $\Omega_{2}$.

Let $\rho$ be a defining function of $\Omega_{2}$. Let $\Sigma_{1}$ and $\Sigma_{2}$ be the sets of weakly pseudoconvex boundary points on $\partial \Omega_{1}$ and $\partial \Omega_{2}$, respectively. Fix a point $p \in \Sigma_{1}$. Suppose $f: \partial \Omega_{1} \rightarrow \partial \Omega_{2}$ is a CR-diffeomorphism. Then since $f$ is a CR-diffeomorphism, $\mathscr{L}_{\rho}\left(f_{*}(L), f_{*}(L)\right)(f(p))=0$ for all $p \in \Sigma_{1}$ and $L \in \mathcal{N}_{p}$. Therefore, $\left.f\right|_{\Sigma_{1}}: \Sigma_{1} \rightarrow \Sigma_{2}$ and $\left.d_{p} f\right|_{\mathcal{N}_{p}}: \mathcal{N}_{p} \rightarrow \mathcal{N}_{f(p)}$ are injective and onto.

Now by Proposition 5.3, if for some $\eta_{2}>1$,

$$
\left(\frac{\eta_{2}}{\eta_{2}-1}\left(\pi_{1,0} \alpha_{\rho} \wedge \pi_{0,1} \alpha_{\rho}\right)-\frac{i}{2} d^{c} \alpha_{\rho}\right)\left(f_{*}(L), \overline{f_{*}(L)}\right) \leq 0
$$

for all $L \in \mathcal{N}_{p}$, then

$$
\left(\frac{\eta_{2}}{\eta_{2}-1}\left(\pi_{1,0} \alpha_{(\rho \circ f)} \wedge \pi_{0,1} \alpha_{(\rho \circ f)}\right)-\frac{i}{2} d^{c} \alpha_{(\rho \circ f)}\right)(L, \bar{L}) \leq 0 .
$$

for all $L \in \mathcal{N}_{p}$. Therefore, this implies $S\left(\Omega_{1}\right) \leq S\left(\Omega_{2}\right)$ by using Theorem 1.1 and the equation (4.5). The same argument for $f^{-1}$ gives $S\left(\Omega_{1}\right) \geq S\left(\Omega_{2}\right)$, and this completes the proof. The statement $D F\left(\Omega_{1}\right)=D F\left(\Omega_{2}\right)$ follows from the same argument as in the Steinness index case.

Acknowledgements: This work is completed while the author visited the University of Wuppertal in May, 2019. The author would like to thank Professor N. Shcherbina, T. Pawlaschyk, T. Harz and H. Herrmann not only for warm hospitality but also for many fruitful conversations. He is also grateful to $\mathrm{M}$. Adachi for his careful reading and comments to imporve this paper.

\section{REFERENCES}

1. H. P. Boas and E. J. Straube, de Rham cohomology of manifolds containing the points of infinite type, and Sobolev estimates for the $\bar{\partial}$-Neumann problem, J. Geom. Anal., 3, no. 3, 225-235. (1993)

2. A. Boggess, CR manifolds and the tangential Cauchy-Riemann complex, Studies in Advanced Mathematics.CRC Press, Boca Raton, FL, 1991. xviii+364 pp. ISBN: 0-8493-7152-X

3. J. P. D'Angelo, Finite type conditions for real hypersurfaces, J. Differential Geom., 14, no. 1, 59-66. (1980)

4. J. P. D'Angelo, Iterated commutators and derivatives of the Levi form, Complex analysis (University Park, Pa., 1986), Lecture Notes in Math., 1268, Springer, Berlin, 103-110. (1987)

5. K. Diederich and J. E. Fornæss, Pseudoconvex Domains: bounded strictly plurisubharmonic exhaustion functions, Invent. Math. 39, no. 2, 129-141. (1977)

6. K. Diederich and J. E. Fornæss, Pseudoconvex Domains: an example with nontrivial neighborhood, Math. Ann. 225, no. 3, 275-292. (1977)

7. B. Liu, The Diederich-Forncess index 1 : for domains of non-trivial index, arXiv:1701.00293v4. (2017) 
8. E. J. Straube, Lectures on the $L^{2}$-Sobolev theory of the $\bar{\partial}$-Neumann problem, ESI Lectures in Mathematics and Physics. (2010)

9. J. Yum, Invariance of Diederich-Fornaes index, arXiv:1702.02301, (2017)

10. J. Yum, On the Steinness index, J. Geom. Anal., 29, no. 2, 1583-1607. (2019)

Republic of KoreA

E-mail address: jihun0224@gmail.com 